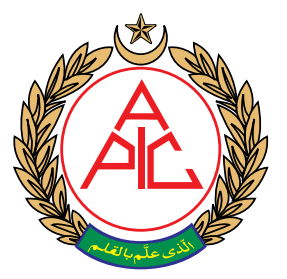

\title{
Sepsis and septic shock
}

\author{
Mayuri Gupta $^{1}$, Shivani Sharma², Pranav Bansal ${ }^{3}$, \\ Priyanka Sehrawat ${ }^{1}$ \\ ${ }^{1}$ Resident, ${ }^{3}$ Professor \& Head \\ Department of Anaesthesiology \& Critical Care, BPS Govt Medical College, Khanpur \\ Kalan, Sonipat, Haryana (India) \\ 2Professor, Department of Physiology, Medical University of the Americas, St. Kitts \\ \& Nevis \\ DOI: 10.35975/apic.v23i4.1185
}

Q 1. Which of the following is recommended for initial resuscitation of the patients with sepsis and septic shock?

A. Treatment should be started after proper evaluation of the patient.

B. For sepsis-induced hypoperfusion, initial resuscitation is initiated with $30 \mathrm{ml} / \mathrm{kg}$ of IV colloids within the first 3 hours.

C. Initial resuscitation is aimed to normalize lactate in patients with elevated lactate levels

D. Initial target mean arterial pressure (MAP) is 50 $\mathrm{mm} \mathrm{Hg}$ in patients with septic shock.

Q 2. Which of the following statement is true regarding blood glucose level management in the patients with septic shock?

A. The approach should target an upper blood glucose level $\leq 180 \mathrm{mg} / \mathrm{dl}$.

B. Blood glucose values to be monitored every $1-2 \mathrm{hr}$ in all the patients.

C. Venous blood is preferred for estimation of blood sugar levels.

D. Intensive insulin therapy improves the survival rate in diabetic patients with sepsis

Q 3. A female patient was presented to the emergency ward and diagnosed with septic shock, following which she was admitted to ICU. The following is true regarding her DVT prophylaxis regimen in ICU:

A. Thromboprophylaxis should not be started unless there is a strong radiological evidence suggestive of thrombosis or embolism.

B. Unfractionated heparin(UFH) should be preferred over low molecular weight heparin (LMWH) for venous thromboembolism prophylaxis in the absence of contraindications to the use of UFH

C. Combined pharmacologic prophylaxis and mechanical prophylaxis is a potential option in critically ill patients with sepsis and septic shock

D. Use of DVT prophylaxis in septic shock can increase the risk of internal hemorrhage

Q 4. Which statement is true regarding ionotropic support in patients with septic shock?

A. Dopamine may be the vasoactive agent of choice if patient presents with tachycardia

B. Low-dose dopamine is recommended for renal protection in such patients

C. Dobutamine is recommended to treat persistent hypoperfusion despite adequate fluid loading and the use of vasopressor agents

D. Epinephrine should be added after norepinephrine with the intent of raising MAP to the target levels.

Q 5. Which of the following is incorrect regarding the management of the patient with sepsis and septic shock?

A. RBC transfusion is recommended at hemoglobin concentration of $<7.0 \mathrm{~g} / \mathrm{dL}$ in adults in the absence of extenuating factors like myocardial ischemia, severe hypoxemia, or acute hemorrhage

B. Clotting abnormalities should be corrected with fresh frozen plasma (FFP) even in the absence of bleeding to prevent hemorrhage

C. Routine use of antithrombin and heparin is not recommended

D. Routine use of IV hydrocortisone in early sepsis is not recommended

Q 6. Q 4. An alcoholic young male admitted to ICU for the management of acute pancreatitis. After 2 days of ICU admission, patient developed fever, hypotension and tachypnoea. Blood investigations revealed raised white blood cells. Which of the following statement is true regarding initial resuscitation of the above patient?

A. Colloids should be the first choice as long as 
hemodynamic factors continue to improve in this patient.

B. Crystalloids along with albumin can be used for initial resuscitation and subsequent intravascular volume replacement

C. Gelatins should be the fluid of choice for resuscitation

D. Use of hydroxyethyl starches (HESs) and gelatin is recommended over albumin for intravascular volume replacement as these have high molecular weight as compare to albumin.

Q 7. Which of the following recommendation is incorrect regarding initiation of antimicrobial therapy for sepsis and septic shock?

A. Administration of IV antimicrobials should be initiated as soon as possible and preferably within 1 hour after recognition

B. Appropriate routine microbiological cultures reports (including blood) must be obtained before starting antimicrobial therapy

C. Empiric broad-spectrum therapy must be initiated with one or more antimicrobials

D. Systemic antimicrobial prophylaxis should be given in patients with severe inflammatory states of non-infectious origin

Q 8: Which of the following is not recommended regarding the mechanical ventilation of the patients with sepsis?

A. Mechanically ventilated sepsis patients be maintained with the head of the bed elevated between $30^{\circ}$ and $45^{\circ}$

B. Use of lower tidal volumes over higher tidal volumes in adult patients with sepsis induced respiratory failure without ARDS

C. Use of high-frequency oscillatory ventilation (HFOV) in adult patients with sepsis-induced ARDS.

D. Use of prone over supine position in adult patients with sepsis-induced $\mathrm{ARDS}$ and $\mathrm{PaO}_{2} / \mathrm{FiO}_{2}$ ratio $<150$

Q 9: Which of the following is recommended as absolute interventional therapy in sepsis?

1. Insertion of arterial catheter for continuous blood sugar monitoring.

2. CVP insertion to guide the hemodynamic assessment and further treatment.

3. PA catheter insertion is routinely indicated in patients with sepsis to guide variable haemodynamic parameters.

4. Placement of post-pyloric feeding tubes in critically ill patients with sepsis or septic shock with feeding intolerance
Q 10: Which of the following is recommended for nutrition in patient with septic shock?

A. Hypocaloric feed

B. Selenium

C. Arginine

D. Glutamine

\section{Explanation}

Ans. 1(C): Sepsis and septic shock are medical emergencies and guidelines recommend that treatment and resuscitation begin immediately. On presentation, $30 \mathrm{ml} / \mathrm{kg}$ of IV crystalloid fluid be given within the first $3 \mathrm{~h}$ for resuscitation from sepsisinduced hypo perfusion (strong recommendation, low-quality evidence). Resuscitation is guided to normalize lactate levels as they are a marker of tissue hypoperfusion (weak recommendation, low-quality evidence). The initial target is to maintain mean arterial pressure (MAP) at $65 \mathrm{mmHg}$ in patients with septic shock requiring vasopressors (strong recommendation, moderate-quality evidence).

Ans. 2(A): Insulin dosing is initiated when two consecutive blood glucose levels are $>180 \mathrm{mg} / \mathrm{dl}$. This approach should target an upper blood glucose level $\leq 180 \mathrm{mg} / \mathrm{dl}$ rather than an upper target blood glucose level $\leq 110 \mathrm{mg} / \mathrm{dl}$. Blood glucose values be monitored every 1-2 $\mathrm{h}$ until glucose values and insulin infusion rates are stable, then every $4 \mathrm{~h}$ thereafter, in patients receiving insulin infusions. Use of arterial blood is preferred to capillary blood for point-of-care testing if patients have arterial catheters (weak recommendation, low-quality evidence). Intensive insulin therapy does not change 28 or 90 -day mortality, and is associated with a higher incidence of hypoglycemia.

Ans. 3(C): Combined pharmacologic prophylaxis and mechanical prophylaxis with intermittent pneumatic compression (IPC) and/or graduated compression stockings (GCS) is a potential option in critically ill patients with sepsis and septic shock, whenever possible (weak recommendation, low-quality evidence). Guidelines recommend LMWH rather than UFH for venous thromboembolism (VTE) prophylaxis in the absence of contraindications to the use of LMWH (strong recommendation, moderatequality evidence).

Ans. 4(D): Nor-epinephrine is the first-choice vasopressor in septic shock (strong recommendation, moderate quality of evidence). Dobutamine may be used in patients who show evidence of persistent hypoperfusion despite adequate fluid loading and the use of vasopressor agents (weak recommendation, low-quality evidence). 
Adding either vasopressin (up to $0.03 \mathrm{U} / \mathrm{min}$ ) (weak recommendation, moderate-quality evidence) or epinephrine (weak recommendation, low-quality evidence) to nor-epinephrine with the intent of raising MAP to target, may also be performed.

Ans. 5(B): Recommendations suggest against the use of fresh frozen plasma to correct clotting abnormalities in the absence of bleeding or planned invasive procedures (weak recommendation, very low-quality evidence).

Guidelines suggest against using IV hydrocortisone for treating septic shock patients if adequate fluid resuscitation and vasopressor therapy are able to restore hemodynamic stability. If this is not achievable, IV hydrocortisone may be started at a dose of $200 \mathrm{mg}$ per day (weak recommendation, lowquality evidence).

Ans. 6(B): Guidelines recommend crystalloids (balanced crystalloids or saline) as the fluid of choice for initial resuscitation and subsequent intravascular volume replacement in patients with sepsis and septic shock (strong recommendation, moderate-quality evidence).

Albumin may be used in addition to crystalloids for initial resuscitation and subsequent intravascular volume replacement in patients with sepsis and septic shock when patients require substantial amounts of crystalloids (weak recommendation, low-quality evidence).

Ans. 7(D): Guidelines recommend against sustained systemic antimicrobial prophylaxis in patients with severe inflammatory states of noninfectious origin (e.g., severe pancreatitis, burn injury).

Ans. 8(C): Bed elevation between $30^{\circ}$ and $45^{\circ}$ decreases the risk of aspiration and prevents the development of ventilator associated pneumonia (VAP) (strong recommendation, low-quality evidence). Guidelines recommend against using high-frequency oscillatory ventilation (HFOV) in adult patients with sepsisinduced ARDS (strong recommendation, moderatequality evidence).

Ans. 9(D): The use of CVP alone to guide fluid resuscitation cannot longer be justified because the ability to predict a response to a fluid challenge when the CVP is within a relatively normal range (8-12 $\mathrm{mm} \mathrm{Hg}$ ) is limited. Guidelines recommend against the routine use of the PA catheter for patients with sepsis-induced ARDS (strong recommendation, high-quality evidence). Guidelines suggest placement of post-pyloric feeding tubes in critically ill patients with sepsis or septic shock with feeding intolerance or who are considered to be at high risk of aspiration (weak recommendation, low-quality evidence).

Ans. 10(A): The sepsis guidelines recommend against the use of selenium, arginine, carnitine, glutamine or omega-3 fatty acids (as an immune supplement) in critically ill patients with sepsis and septic shock (strong recommendation, moderatequality evidence). Early trophic/ hypocaloric or early full enteral feeding should be initiated in critically ill patients with sepsis or septic shock; if trophic/ hypocaloric feeding is the initial strategy, then feeds should be advanced according to patient tolerance.

Reference: Surviving Sepsis Guidelines 2016 and 2018 